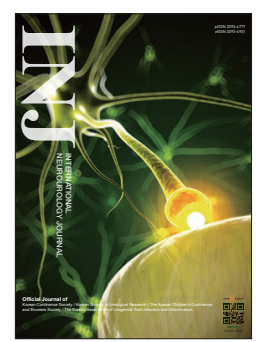

\title{
Applications of Microfluidic Devices for Urology
}

\author{
Se Jik Han ${ }^{1}$, Hun-Kuk Park ${ }^{2,3,4}$, Kyung Sook Kim ${ }^{2,4}$ \\ ${ }^{1}$ Department of Biomedical Engineering, Graduate School, Kyung Hee University, Seoul, Korea \\ ${ }^{2}$ Department of Biomedical Engineering, College of Medicine, Kyung Hee University, Seoul, Korea \\ ${ }^{3}$ Healthcare Industry Research Institute, Kyung Hee University, Seoul, Korea \\ ${ }^{4}$ Program of Medical Engineering, Kyung Hee University, Seoul, Korea
}

Microfluidics is considered an important technology that is suitable for numerous biomedical applications, including cancer diagnosis, metastasis, drug delivery, and tissue engineering. Although microfluidics is still considered to be a new approach in urological research, several pioneering studies have been reported in recent years. In this paper, we reviewed urological research works using microfluidic devices. Microfluidic devices were used for the detection of prostate and bladder cancer and the characterization of cancer microenvironments. The potential applications of microfluidics in urinary analysis and sperm sorting were demonstrated. The use of microfluidic devices in urology research can provide high-throughput, high-precision, and low-cost analyzing platforms.

Keywords: Microfluidics; Urology; Prostatic Neoplasms; Urinary Bladder Neoplasms; Urine

- Conflict of Interest: No potential conflict of interest relevant to this article was reported.

\section{INTRODUCTION}

Microfluidics is the science and technology that deals with fluids usually in the range of microliters $\left(10^{-6}\right)$ to picoliters $\left(10^{-12}\right)$. Since microfluidics typically deals with fluids in the microliter scale, it has several advantages, including low consumption of samples, short analysis time, and high sensitivity [1-4]. The portability and fast processing speed of microfluidic devices also allows for in situ and real-time analysis.

A conventional microfluidic device consists of microchannels molded in a polymer. Polydimethylsiloxane (PDMS) is commonly used for molding microfluidic chips because it is a transparent and biocompatible elastomer. The fabricating process of PDMS-based microfluidic devices, which is based on soft lithography, consists of the following steps: (1) master fab- rication steps, including spin coating of a photoresist film, exposure, and development to form the mold on a silicon substrate; (2) device fabrication steps, including pouring the PDMS on the master, punching holes with a biopsy punch, and bonding the PDMS structure to glass. Recently, paper-based microfluidic devices have been proposed as cheap, portable, and disposable devices [5-7].

Ever since this emerging discipline was introduced in the early 1990s, microfluidics has grown rapidly in the field of biomedical applications [8,9]. Microfluidic devices are very useful tools for molecular separation, biochemical assays, drug screening, chromatography, and migration assays [10-14]. Lap-on-chip and organ-on-chip based on microfluidic devices have also been widely used for high-throughput screening applications $[15,16]$.

Microfluidic devices have not been widely used in urological
Corresponding author: Kyung Sook Kim (iD http://orcid.org/0000-0003-3572-1169 Department of Biomedical Engineering, College of Medicine, Kyung Hee University, 26 Kyungheedae-ro, Dongdaemun-gu, Seoul 02447, Korea E-mail: moosou94@khu.ac.kr / Tel: +82-2-961-9501 / Fax: +82-2-6008-5535 Submitted: March 2, 2017 / Accepted after revision: March 12, 2017 cc) (i) This is an Open Access article distributed under the terms of the Creative Commons Attribution Non-Commercial License (http://creativecommons.org/licenses/by-nc/4.0/) which permits unrestricted non-commercial use, distribution, and reproduction in any medium, provided the original work is properly cited. 
research yet. However, some pioneering studies have been reported for bladder and prostate cancer detection, urine analysis, and sperm characterization. In this review, we will summarize research works that use microfluidics and discuss its application in urology.

\section{MICROFLUIDIC PLATFORM FOR UROLOGICAL RESEARCHES}

\section{Screening for Prostate and Bladder Cancer}

Microfluidics has been considered a promising tool to analyze cancer cells and tumor function owing to its high sensitivity, high throughput, and less material consumption. Microfluidic devices offer various microenvironments for cell culture, from 2-dimensional to complex 3-dimensional systems, and the complex coculture system $[17,18]$. Various microfluidic platforms have been developed to understand cancer cell migration and metastasis under various conditions, such as varying chemical gradients and mechanical constraints (Fig. 1) [19]. A microfluidic device to detect metastatic cancer cells based on their size and deformability was also fabricated [20].
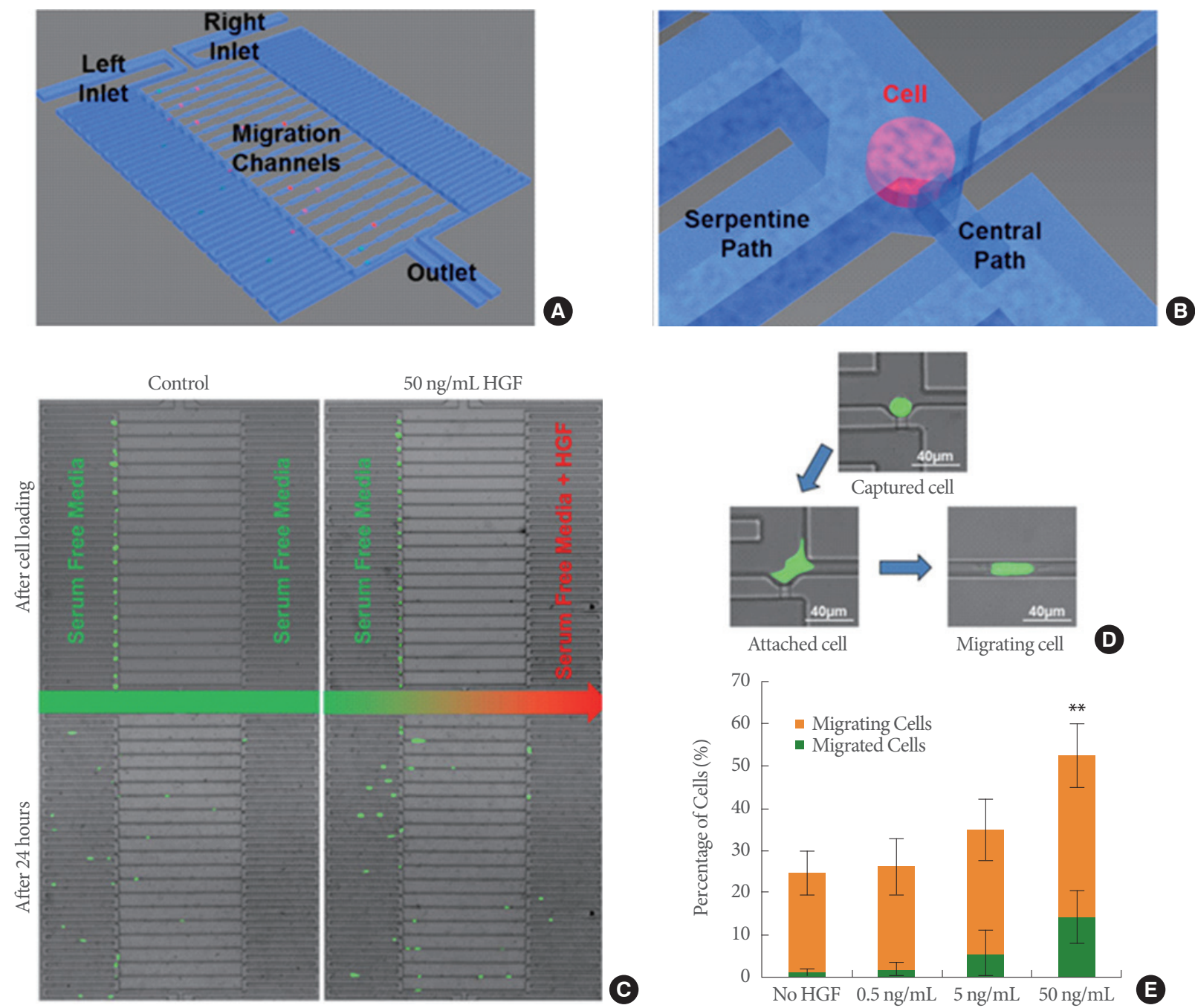

Fig. 1. (A) A schematic illustration of microfluidic device for single-cell migration. (B) Enlarged schematics of cell capture site in the device. (C) The single-cell (SKOV3) migration assay depending on hepatocyte growth factor (HGF). The migration of SKOV3 with and without (control) HGF was observed for 24 hours. The 2 images in upper row shows the distribution of cells after loading, and that of lower row shows the distribution after 24 hours. (D) The cell migrated in three steps of captured, attached, and migrating. (E) Result of the migration assay as a function of HGF concentration. The SKOV3 cells migrated more as the HGF concentration increases. Reprinted from Chen et al. Sci Rep 2015;5:9980 [19], with permission of Nature Publishing Group. 
A microfluidic device has been developed for prostate and bladder cancer research for various procedures, including biomarker detection, characterization of cancer cells in various microenvironments, and circulating tumor detection. Prostate cancer is the most common type of cancer among males and the sixth most common cause of death in males [21]. A prostatic-specific antigen (PSA), which is one of the available biomarkers of prostate cancer, is widely used for the screening of prostate cancer. However, the conventional PSA test has drawbacks, such as excessive sample consumption, restrictive control of the analytes in the reaction chamber, and lack of multiplexing capabilities [22]. Therefore, the use of a microfluidic device has been considered an efficient approach to overcome these drawbacks. Madaboosi et al. [23] fabricated a PSA detection system based on a microfluidic immunoassay (Fig. 2). The microfluidic enzyme-linked immunosorbent assay was integrated with photodetectors to sense the free isoform of the PSA with labeled antibodies by using all 3 detection modes. This integrated system showed enhanced reliability in signal acquisition and refined sensitivity even for low detection limits. Chiriacò et al. [24] introduced a PSA microfluidic sensing platform integrated with electrochemical impedance spectroscopy. This platform allowed for quick screening and contemporary detection of free and total PSA using 2 different antibodies that are immobilized on a single chip.

Bladder cancer is a common urologic cancer that has the highest recurrence rate among all other malignancies [25]. The microfluidic-based microenvironment is a good model to investigate the tumor growth, metastasis, and dynamic interplay between the cells. The mitochondrial-related protein expression in the bladder cancer cells was investigated in coculture conditions using a microfluidic chip $[17,18]$. A chip for the coculture of bladder tumor cells and fibroblasts was composed of 2 cell culture pools for human skin fibroblasts and the human bladder tumor cells. The pools were connected using microchannels and peripheral perfusion channels. Using this coculture system, it was observed that the high-energy metabolites transferred to adjacent tumor cells in a specified direction. To study the effects of the microenvironment on bladder cancer, the microfluidic device based on 4 types of cells coculture system was suggested [18]. This system consisted of perfusion equipment, matrigel channel units, a medium channel, and four indirect contact culture chambers in which the 4 types of cells (stromal cells, fibroblasts, endothelial cells, and macrophages) could be cultured simultaneously.

\section{Fast and Reliable Urine Analysis}

Clinical analysis of urine has been used to diagnose several dis-

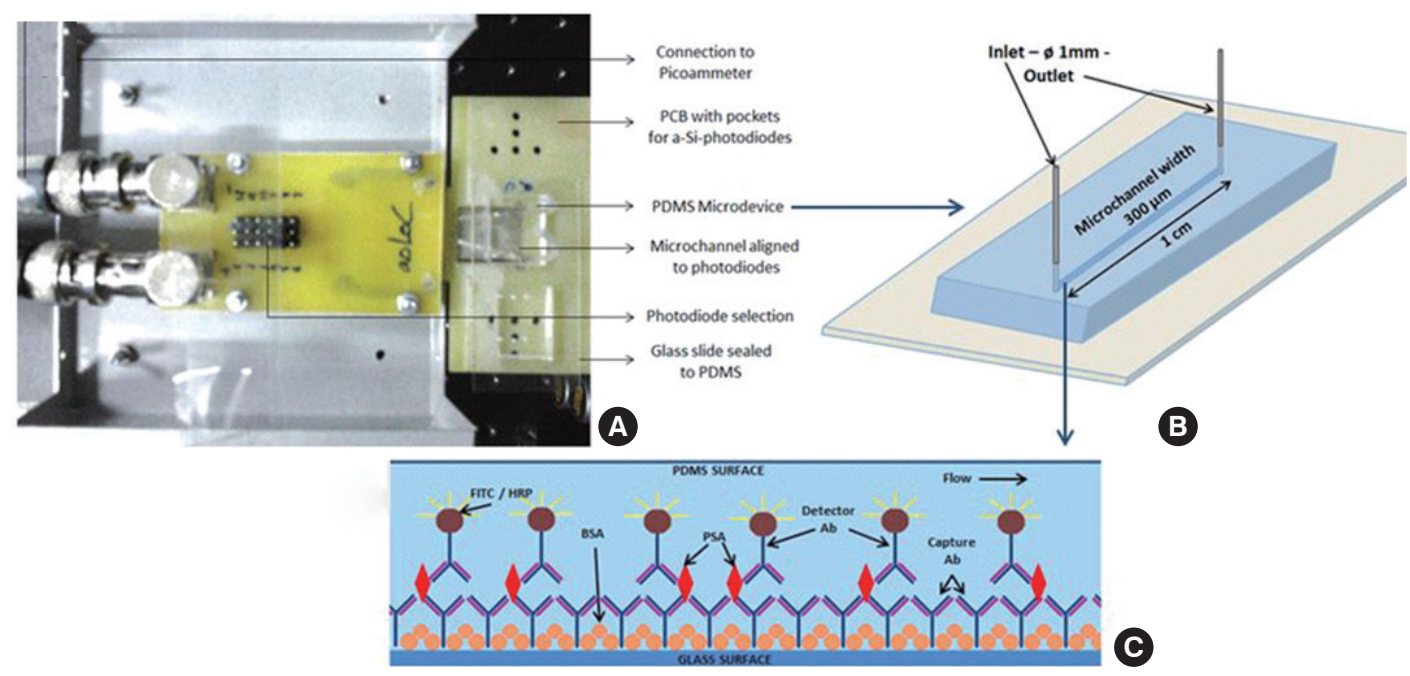

Fig. 2. (A) Microfluidic-enzyme-linked immunosorbent assay (ELISA) system for the sensing of prostate cancer biomarkers using integrated photodiodes. (B) A schematic illustration of microfluidic channel for forming micro-ELISA $(1 \mathrm{~cm} \times 300 \mu \mathrm{m})$ and inlet/outlet $(0.1 \mathrm{~cm})$. (C) A schematics of prostatic-specific antigen (PSA) sensing by combination of microfluidics and immunoassays. PCB, printed circuit board; PDMS, polydimethylsiloxane; FITC, fluorescein isothiocyanate; HRP, horse-radish peroxidase; BSA, bovine serum albumin; Ab, antibody. Reprinted from Madaboosi et al. Sensor, IEEE 2014 proceeding [23], with permission of Institute of Electrical and Electronics Engineers. 
eases. The changes in urine composition, such as the changes in the levels of glucose, albumin, creatinine, and uric acid, may provide information about organ dysfunctions, drug abuse, and exposure to chemicals and toxins [26]. Conventional methods to examine urine samples are physical (color, odor, etc.), biochemical, and microscopic examinations. Microfluidic devices are typically used in the manipulation of fluids with a small quantity of sample, under a precisely controlled flow rate. Therefore, they are very useful devices for urine sample pretreatments and sensing urinary molecules. Miyaguchi et al. [27] developed a microchip for liquid-liquid extraction as a sample pretreatment device prior to carrying out gas chromatography analysis of the amphetamine-type stimulants in the urine (Fig. $3 \mathrm{~A})$. The interface between the organic solvent and alkalized urine was stabilized by using the microchannels modified by a capillary-restricted modification method. Microfluidic devices have been used for the detection of urinary macromolecules, including protein, virus, and bacteria. Kaigala et al. [28] demonstrated the cost-effective detection of the BK virus (viral load) from nonpretreated urine samples using microfluidic devices. A detection sensitivity of as low as 1-2 viral copies was obtained by the integration of a small volume genetic amplification (polymerase chain reaction). Microfluidic devices for urinary micromolecules, including creatinine, galactose, and inor- ganic ions have also been demonstrated by several groups. For analyzing urinary creatinine, a microfluidic system based on a 2-point alkaline picrate kinetic reaction was developed (Fig. 3B) [29]. The microchip was connected to 2 syringes for delivering the samples, and it was connected to a miniature fiber optic spectrophotometer for measuring the absorption light at 510 $\mathrm{nm}$. This system exhibited good reproducibility and a high correlation with the conventional spectrophotometric method. The integration of microfluidic devices with different approaches, including electrophoresis, optics, and immunoassays, was successfully used for detecting drugs and metabolites in urine samples [30,31]. Caffeine and theophylline could be effectively separated using the microchannel electrophoresis integrated with electrochemical detection, and illicit drugs were detected with a high accuracy using the microfluidic device with an ultraviolet detection function.

\section{Sorting Sperm}

Male infertility is closely related to the motility of sperm. Many microfluidic systems have been proposed for quantifying sperm quality and sorting sperm by their motility. According to the principle, the microfluidic device for sperm sorting can be categorized into the following 3 groups: passively driven devices, flow-driven devices, and combined devices for thermotaxis and
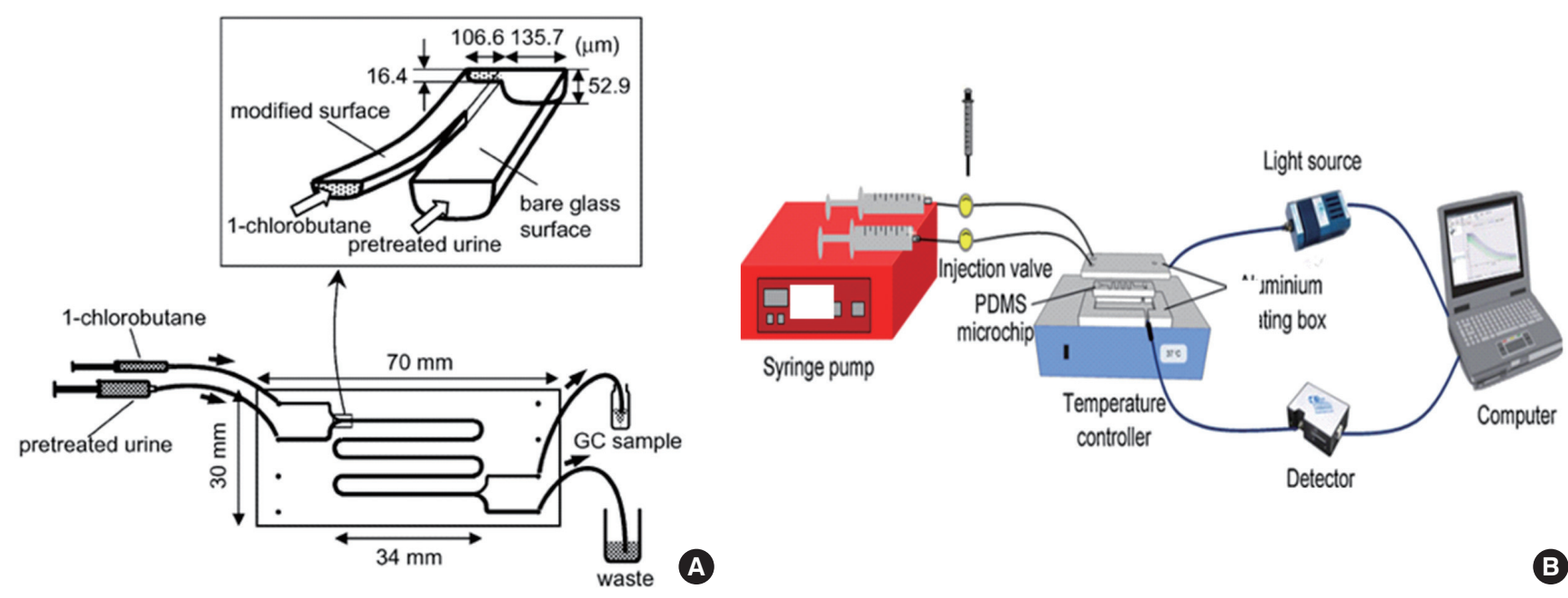

Fig. 3. (A) Illustration of the microchip-based liquid-liquid extraction system for the gas-chromatography analysis of amphetamine in urine. The long microchannel (180-mm length) with 2 inlets and outlets respectively were fabricated on a thick Pyrex glass plate. PDMS, polydimethylsiloxane. Reprinted from Miyaguchi et al. J Chromatogr A 2006;1129:105-10 [27], with permission of Elsevier B.V. (B) A schematic illustration of the portable microfluidic system for determination creatinine in urine. The microfluidic system is consisted of 2 injection valves, aluminum heating box, temperature controller, light source, and detector. Reprinted from Songjaroen et al. Anal Chim Acta 2009;647:78-83 [29], with permission of Elsevier B.V. 
chemotaxis [32]. The principle of passively driven devices is that motile sperm can be collected within a specific period of time in the outlet of the microfluidic channels, while less-motile sperm are left behind in the microchannels [33]. In flow-driven devices, sperm encounters a flow in a direction that is opposite to its movement [34]. The sperm are segregated into different channels depending on their motility and are counted thereafter. The combined microfluidic device with a chemoattractant was fabricated with a straight channel connected to a bi-branch channel [35]. The sperm motility under the chemical gradient was monitored, and the chemotaxis was analyzed by using the ratio of the sperm collected in different branches.

\section{CONCLUSIONS}

Although a microfluidic system has many advantages, it is still a relatively new technology in the field of urology research. Recently, some successful implementations of microfluidic devices have been demonstrated for cancer diagnosis, urine analysis, and sperm sorting. However, more promising microfluidic systems and research works are required for such systems to be applied in the clinic. Therefore, it is important to continue the development of this new technology in cooperation with the relevant users.

\section{REFERENCES}

1. Whitesides GM. The origins and the future of microfluidics. Nature 2006;442:368-73.

2. El-Ali J, Sorger PK, Jensen KF. Cells on chips. Nature 2006;442:40311.

3. Kose AR1, Fischer B, Mao L, Koser H. Label-free cellular manipulation and sorting via biocompatible ferrofluids. Proc Natl Acad Sci U S A 2009;106:21478-83.

4. Feng X, Du W, Luo Q, Liu BF. Microfluidic chip: next-generation platform for systems biology. Anal Chim Acta 2009;650:83-97.

5. Martinez AW, Phillips ST, Whitesides GM, Carrilho E. Diagnostics for the developing world: microfluidic paper-based analytical devices. Anal Chem 2010;82:3-10.

6. Osborn JL, Lutz B, Fu E, Kauffman P, Stevens DY, Yager P. Microfluidics without pumps: reinventing the T-sensor and $\mathrm{H}$-filter in paper networks. Lab Chip 2010;10:2659-65.

7. Lu Y, Shi W, Qin J, Lin B. Fabrication and characterization of paperbased microfluidics prepared in nitrocellulose membrane by wax printing. Anal Chem 2010;82:329-35.
8. Xia Y, Whitesides GM. Soft lithography. Annu Rev Mater Sci 1998;28:153-84.

9. Duffy DC, McDonald JC, Schueller OJ, Whitesides GM. Rapid prototyping of microfluidic systems in poly(dimethylsiloxane). Anal Chem 1998;70:4974-84.

10. Soper SA, Wang W. Bio-MEMS: technologies and applications. BocaRaton: CRC Press; 2007.

11. Tian WC, Finehout E, editors. Microfluidics for biological applications. New York: Springer; 2008.

12. Abhyankar VV, Toepke MW, Cortesio CL, Lokuta MA, Huttenlocher A, Beebe DJ. A platform for assessing chemotactic migration within a spatiotemporally defined 3D microenvironment. Lab Chip 2008;8:1507-15.

13. Campbell CJ, Grzybowski BA. Microfluidic mixers: from microfabricated to self-assembling devices. Philos Trans A Math Phys Eng Sci 2004;362:1069-86.

14. Diao J, Young L, Kim S, Fogarty EA, Heilman SM, Zhou P, et al. A three-channel microfluidic device for generating static linear gradients and its application to the quantitative analysis of bacterial chemotaxis. Lab Chip 2006;6:381-8.

15. Temiz Y, Lovchik RD, Kaigala GV, Delamarche E. Lab-on-a-chip devices: How to close and plug the lab? Microelectron Eng 2015; 132:156-75.

16. Bhatia SN, Ingber DE. Microfluidic organs-on-chips. Nat Biotechnol 2014;32:760-72.

17. Xu XD, Shao SX, Cao YW, Yang XC, Shi HQ, Wang YL, et al. The study of energy metabolism in bladder cancer cells in co-culture conditions using a microfluidic chip. Int J Clin Exp Med 2015;8: 12327-36.

18. Liu PF, Cao YW, Zhang SD, Zhao Y, Liu XG, Shi HQ, et al. A bladder cancer microenvironment simulation system based on a microfluidic co-culture model. Oncotarget 2015;6:37695-705.

19. Chen YC, Allen SG, Ingram PN, Buckanovich R, Merajver SD, Yoon E. Single-cell migration chip for chemotaxis-based microfluidic selection of heterogeneous cell populations. Sci Rep 2015;5: 9980.

20. Islam M, Asghar W, Kim YT, Iqbal SM. Cell elasticity-based microfluidic label-free isolation of metastatic tumor cells. Br J Med Med Res 2014;4:2129-40.

21. Velonas VM, Woo HH, dos Remedios CG, Assinder SJ. Current status of biomarkers for prostate cancer. Int J Mol Sci 2013;14:11034-60.

22. Shariat SF, Karam JA, Margulis V, Karakiewicz PI. New bloodbased biomarkers for the diagnosis, staging and prognosis of prostate cancer. BJU Int 2008;101:675-83.

23. Madaboosi N, Pedrosa CR, Reis MF, Soares RR, Chu V, Conde JP. 
Microfluidic ELISA for sensing of prostate cancer biomarkers using integrated a-Si:H p-i-n photodiodes. In: Sensor, IEEE 2014 proceeding; 2014 Nov 2-5; Valencia, Spain. Piscataway (NJ): Institute of Electrical and Electronics Engineers; 2014.

24. Chiriacò MS, Primiceri E, Montanaro A, de Feo F, Leone L, Rinaldi $\mathrm{R}$, et al. On-chip screening for prostate cancer: an EIS microfluidic platform for contemporary detection of free and total PSA. Analyst 2013;138:5404-10.

25. Jemal A, Bray F, Center MM, Ferlay J, Ward E, Forman D. Global cancer statistics. CA Cancer J Clin 2011;61:69-90.

26. Lin CC, Tseng CC, Chuang TK, Lee DS, Lee GB. Urine analysis in microfluidic devices. Analyst 2011;136:2669-88.

27. Miyaguchi H, Tokeshi M, Kikutani Y, Hibara A, Inoue H, Kitamori T. Microchip-based liquid-liquid extraction for gas-chromatography analysis of amphetamine-type stimulants in urine. J Chromatogr A 2006;1129:105-10.

28. Kaigala GV, Huskins RJ, Preiksaitis J, Pang XL, Pilarski LM, Backhouse CJ. Automated screening using microfluidic chip-based PCR and product detection to assess risk of BK virus-associated nephropathy in renal transplant recipients. Electrophoresis 2006;27: 3753-63.
29. Songjaroen T, Maturos T, Sappat A, Tuantranont A, Laiwattanapaisal W. Portable microfluidic system for determination of urinary creatinine. Anal Chim Acta 2009;647:78-83.

30. Zhang QL, Lian HZ, Wang WH, Chen HY. Separation of caffeine and theophylline in poly(dimethylsiloxane) microchannel electrophoresis with electrochemical detection. J Chromatogr A 2005; 1098:172-6.

31. Qiang W, Zhai C, Lei J, Song C, Zhang D, Sheng J, et al. Disposable microfluidic device with ultraviolet detection for highly resolved screening of illicit drugs. Analyst 2009;134:1834-9.

32. Knowlton SM, Sadasivam M, Tasoglu S. Microfluidics for sperm research. Trends Biotechnol 2015;33:221-9.

33. Cho BS, Schuster TG, Zhu X, Chang D, Smith GD, Takayama S. Passively driven integrated microfluidic system for separation of motile sperm. Anal Chem 2003;75:1671-5.

34. Chen YA, Huang ZW, Tsai FS, Chen CY, Lin CM, Wo AM. Analysis of sperm concentration and motility in a microfluidic device. Microfluid Nanofluidics 2011;10:59-67.

35. Xie L, Ma R, Han C, Su K, Zhang Q, Qiu T, et al. Integration of sperm motility and chemotaxis screening with a microchannelbased device. Clin Chem 2010;56:1270-8. 